\title{
SOME TRACE INEQUALITIES FOR DISCRETE GROUPS OF MÖBIUS TRANSFORMATIONS
}

\author{
CHUN CAO
}

(Communicated by Albert Baernstein II)

\begin{abstract}
We show that if $\langle A, B\rangle$ is discrete where $A, B \in \mathrm{SL}(2, \mathbb{C})$ and if $\operatorname{tr}\left(A B A^{-1} B^{-1}\right) \neq 2, \operatorname{tr}\left(A B A B^{-1}\right) \neq 2$, and $\left|\operatorname{tr}^{2}(A)-4\right| \leq 2(\cos (2 \pi / 7)+$ $\cos (\pi / 7)-1)=1.0489 \ldots$, then
\end{abstract}

$$
\left|\operatorname{tr}\left(A B A^{-1} B^{-1}\right)-2\right| \geq 2-2 \cos (\pi / 7)=0.198 \ldots .
$$

It follows from above that if $\langle X, Y\rangle$ is discrete with $\operatorname{tr}(X)=\operatorname{tr}(Y) \neq 0$ and $\operatorname{tr}\left(X Y X^{-1} Y^{-1}\right) \neq 2$, then

$$
\left|\operatorname{tr}\left(X Y X^{-1} Y^{-1}\right)-2\right| \geq 2-2 \cos (\pi / 7)=0.198 \ldots .
$$

Both inequalities are sharp.

\section{INTRODUCTION}

A subgroup of $\operatorname{SL}(2, \mathbb{C})$ is said to be discrete if it does not contain any convergent sequences of distinct elements. There is an important necessary condition due to Jørgensen [8] for a two-generator group to be discrete.

If $A$ and $B$ generate a discrete subgroup of $\mathrm{SL}(2, \mathbb{C})$, then

$$
\left|\operatorname{tr}^{2}(A)-4\right|+\left|\operatorname{tr}\left(A B A^{-1} B^{-1}\right)-2\right| \geq 1,
$$

unless $B A B^{-1} \in\left\{A, A^{-1}\right\}$, in which case the subgroup is elementary.

The commutator trace is not uniformly bounded away from 2 . In other words, there does not exist a positive real number $K$ such that $\mid \operatorname{tr}\left(A B A^{-1} B^{-1}\right)-$ $2 \mid \geq K$ holds whenever $A$ and $B$ generate a nonelementary discrete group [10]. However, Jørgensen has shown that

$$
\left|\operatorname{tr}\left(X Y X^{-1} Y^{-1}\right)-2\right|>0.125
$$

if $X$ and $Y$ with equal traces generate a nonelementary discrete subgroup [11]. Inequality (1.2) was sharpened by Gehring and Martin [4] to give

$$
\left|\operatorname{tr}\left(X Y X^{-1} Y^{-1}\right)-2\right|>0.193,
$$

and they conjectured that

$$
\left|\operatorname{tr}\left(X Y X^{-1} Y^{-1}\right)-2\right| \geq 2-2 \cos (\pi / 7)=0.198 \ldots
$$

Received by the editors June 13, 1994.

1991 Mathematics Subject Classification. Primary 30F40.

Research supported in part by grants from the National Science Foundation. 
if $\operatorname{tr}\left(X Y X^{-1} Y^{-1}\right) \neq 2, \operatorname{tr}^{2}(X)=\operatorname{tr}^{2}(Y) \neq 0$, and $X, Y$ generate a discrete subgroup.

We show that $\operatorname{tr}\left(A B A^{-1} B^{-1}\right)-2$ is bounded away from zero if $\left|\operatorname{tr}^{2}(A)-4\right|$ is not too large. In particular, if $A$ and $B$ generate a discrete group and if $\operatorname{tr}\left(A B A^{-1} B^{-1}\right) \neq 2, \operatorname{tr}\left(A B A B^{-1}\right) \neq 2$, and

$$
\left|\operatorname{tr}^{2}(A)-4\right| \leq 2(\cos (2 \pi / 7)+\cos (\pi / 7)-1)=1.0489 \ldots,
$$

then

$$
\left|\operatorname{tr}\left(A B A^{-1} B^{-1}\right)-2\right| \geq 2-2 \cos (\pi / 7)=0.198 \ldots .
$$

We show in section 4 that the conjecture (1.4) of Gehring and Martin is true. We then apply these results to get many other inequalities.

\section{Notation}

Let $M$ denote the group of all Möbius transformations of the extended complex plane $\overline{\mathbb{C}}=\mathbb{C} \cup\{\infty\}$. We associate with each

$$
f=\frac{a z+b}{c z+d} \in \mathbb{M}, \quad a d-b c=1,
$$

the matrix

$$
A=\left(\begin{array}{ll}
a & b \\
c & d
\end{array}\right) \in \mathrm{SL}(2, \mathbb{C})
$$

and set $\operatorname{tr}(f)=\operatorname{tr}(A)$, where $\operatorname{tr}(A)$ denotes the trace of $A$. Note that $\operatorname{tr}(f)$ is defined up to sign.

For each $f$ and $g$ in $\mathbb{M}$ we let $[f, g]$ denote the multiplicative commutator $f g f^{-1} g^{-1}$. We call the three complex numbers

$$
\beta(f)=\operatorname{tr}^{2}(f)-4, \quad \beta(g)=\operatorname{tr}^{2}(g)-4, \quad \gamma(f, g)=\operatorname{tr}([f, g])-2
$$

the parameters of the two-generator group $\langle f, g\rangle$ and write

$$
\operatorname{par}(\langle f, g\rangle)=(\gamma(f, g), \beta(f), \beta(g)) .
$$

These parameters are independent of the choice of representative matrices for $f$ and $g$, and they determine $\langle f, g\rangle$ up to conjugacy whenever $\gamma(f, g) \neq 0$ [2]. But see [1] for three-generator groups. Note that $\gamma(f, g) \neq 0$ if and only if $f$ and $g$ do not have a common fixed point in $\overline{\mathbb{C}}$.

\section{A SHARP BOUND}

3.1. Theorem. If $\langle f, g\rangle$ is discrete with $\gamma(f, g) \neq 0$ and $\beta(g) \neq-4$ and if

$$
|\beta(f)| \leq c=2(\cos (2 \pi / 7)+\cos (\pi / 7)-1)=1.0489 \ldots,
$$

then

$$
|\gamma(f, g)| \geq d=2-2 \cos (\pi / 7)=0.198 \ldots .
$$

Inequality (3.3) holds when $\langle f, g\rangle$ is the $(2,3,7)$ triangle group with parameters $\beta(f)=\beta(g)=c, \gamma(f, g)=-d$.

Proof. Set

$$
m=\inf \{|\gamma(f, g)|:|\beta(f)| \leq c, \gamma(f, g) \neq 0, \beta(g) \neq-4,\langle f, g\rangle \text { discrete }\} .
$$

Suppose that $m<d=2-2 \cos (\pi / 7)$. We will obtain a contradiction. 
For each $\epsilon$ with $0<\epsilon<\frac{1}{2}(d-m)$, there exist $f, g$ such that

$$
|\gamma(f, g)|<m+\epsilon, \quad|\beta(f)| \leq c, \quad \beta(g) \neq-4 .
$$

If $\gamma(f, g)=\beta(f)$, then $f$ is of order 3,4 or 6 by [4, Lemma 2.10], and hence $|\gamma(f, g)|=|\beta(f)| \geq 1$. This contradicts the assumption that $|\gamma(f, g)|<d$. Thus we may assume that $\gamma(f, g) \neq \beta(f)$. By [7, Lemma 2.29], there exists an elliptic $h$ of order 2 such that $\langle f, h\rangle$ is discrete with $\gamma(f, h)=\gamma(f, g)$.

We will use the following two formulae for $F$ and $G$ in $M$ :

$$
\begin{aligned}
\gamma\left([F, G], F[F, G] F^{-1}\right) & =-\gamma^{2}(F, G)(\gamma(F, G)-\beta(F))(\beta(F)+4), \\
\beta([F, G]) & =\gamma(F, G)(\gamma(F, G)+4) .
\end{aligned}
$$

We define

$$
f_{0}=f, \quad g_{0}=h, \quad f_{n+1}=\left[f_{n}, g_{n}\right], \quad g_{n+1}=f_{n}\left[f_{n}, g_{n}\right] f_{n}^{-1} .
$$

If $\left|\beta\left(f_{2}\right)\right| \leq c$ and $\gamma\left(f_{2}, g_{2}\right) \neq 0$, then by the definition of $m,\left|\gamma\left(f_{2}, g_{2}\right)\right| \geq m$. Let $\gamma=\gamma(f, h), \beta=\beta(f)$. We have

$$
\begin{aligned}
\left|\gamma\left(f_{1}, g_{1}\right)\right| & =\left|-\gamma^{2}(\gamma-\beta)(\beta+4)\right| \leq d^{2}(d+c)(c+4)=d(d+c), \\
\left|\beta\left(f_{2}\right)\right| & =\left|\gamma\left(f_{1}, g_{1}\right)\left(\gamma\left(f_{1}, g_{1}\right)+4\right)\right| \leq c .
\end{aligned}
$$

So, $\left|\gamma\left(f_{2}, g_{2}\right)\right| \geq m$, that is,

$$
\left|\gamma^{5}(\gamma-\beta)^{2}(\beta+4)^{2}(\gamma(\gamma-\beta)(\beta+4)+\gamma+4)(\gamma+2)^{2}\right| \geq m .
$$

Set $p(\gamma, \beta)=\gamma^{4}(\gamma-\beta)^{2}(\beta+4)^{2}(\gamma(\gamma-\beta)(\beta+4)+\gamma+4)(\gamma+2)^{2}$.

Consider one of the polynomials in [7, Lemma 2.1],

$$
\gamma\left(f, h f^{-1} h^{-1} f h f h^{-1} f^{-1} h\right)=\gamma\left(\gamma^{2}-(\beta-1) \gamma-(\beta-1)\right)^{2} .
$$

We consider three cases.

Case 1. Suppose that $\gamma^{2}-(\beta-1) \gamma-(\beta-1)=0$. Then

$$
\beta=1+\frac{\gamma^{2}}{1+\gamma}, \quad p(\gamma, \beta)=\frac{\gamma^{4}\left(5+5 \gamma+\gamma^{2}\right)^{2}(\gamma+2)^{4}}{(1+\gamma)^{6}} .
$$

Let

$$
q(z)=\frac{z^{4}\left(5+5 z+z^{2}\right)^{2}(z+2)^{4}}{(1+z)^{6}} .
$$

It is easy to check that

$$
\max _{|z|=d}|q(z)|=1,
$$

and this maximum is obtained when $z=-d$.

Since $m+\frac{1}{2}(d-m)<d$, there is a constant $a$ such that

$$
\max _{|z| \leq m+\frac{1}{2}(d-m)}|q(z)| \leq a<1 .
$$

By (3.4),

$$
(m+\epsilon) a \geq|\gamma p(\gamma, \beta)| \geq m .
$$

Thus

$$
\epsilon \geq(1-a) m / a .
$$

By [4, Lemma 3.25], $m>0.193$. Taking $\epsilon<\min \{(d-m) / 2,(1-a) m / a\}$ will give a contradiction. 
Case 2. Suppose that $\beta\left(h f^{-1} h^{-1} f h f h^{-1} f^{-1} h\right)=-4$. If $\beta=0$, then $|\gamma| \geq 1$ by the Shimizu-Leutbecher inequality [13, II.C.5]. This contradicts the assumption that $|\gamma|<d$. If $\beta \neq 0$, then we may assume that $f$ and $h$ are represented by

$$
A=\left(\begin{array}{cc}
u & 0 \\
0 & 1 / u
\end{array}\right), \quad B=\left(\begin{array}{ll}
e_{11} & e_{12} \\
e_{21} & e_{22}
\end{array}\right) .
$$

Thus $\beta=(u-1 / u)^{2}, \gamma=-e_{12} e_{21}(u-1 / u)^{2}$. Elementary calculations show that

$$
\begin{aligned}
B A^{-1} & B^{-1} A B A B^{-1} A^{-1} B \\
\quad= & \left(\begin{array}{cc}
e_{11}\left((\gamma+1)^{2}-\gamma u^{-2}\right) & e_{12}\left(\gamma^{2}-(\beta-1) \gamma-(\beta-1)\right) \\
e_{21}\left(\gamma^{2}-(\beta-1) \gamma-(\beta-1)\right) & e_{22}\left((\gamma+1)^{2}-\gamma u^{2}\right)
\end{array}\right) .
\end{aligned}
$$

Since $h f^{-1} h^{-1} f h f h^{-1} f^{-1} h$ is of order two,

$$
e_{11}\left((\gamma+1)^{2}-\gamma u^{-2}\right)+e_{22}\left((\gamma+1)^{2}-\gamma u^{2}\right)=0 .
$$

Notice that $e_{11}+e_{22}=0$ ( $h$ is of order two). If $e_{11} \neq 0$, then (3.5) implies that $\beta(\beta+4)=0$, a contradiction. If $e_{11}=0$, then $e_{12} e_{21}=-1$. Hence $\gamma=\beta$, another contradiction.

Case 3. Suppose that Case 1 and Case 2 do not hold. By the definition of $m$,

$$
\left|\gamma\left(f, h f^{-1} h^{-1} f h f h^{-1} f^{-1} h\right)\right|=\left|\gamma\left(\gamma^{2}-(\beta-1) \gamma-(\beta-1)\right)^{2}\right| \geq m .
$$

It follows that

$$
|\beta-1|>\frac{1}{1+d}\left(\sqrt{m /(m+\epsilon)}-d^{2}\right)
$$

Let

$$
\beta=\rho e^{i \theta}, \quad-\pi<\theta \leq \pi, \quad s=\frac{1}{1+d}\left(\sqrt{m /(m+\epsilon)}-d^{2}\right) .
$$

From (3.6), we have

$$
\cos \theta \leq \frac{1}{2 \rho}\left(1+\rho^{2}-s^{2}\right) .
$$

We apply Jørgensen's inequality (1.1) to get $\rho=|\beta| \geq 1-d$. Thus $1-d \leq$ $\rho \leq c$. It follows from (3.7) that

$$
\cos \theta \leq \frac{1}{2 c}\left(1+c^{2}-s^{2}\right)
$$

By taking sufficiently small $\epsilon$, we obtain $|\theta|>0.8$. We now expand $p(\gamma, \beta)$ to get

$$
p(\gamma, \beta)=\sum_{n=4}^{10} p_{n}(\beta) \gamma^{n},
$$

where $p_{n}(z)=(z+4)^{2} q_{n}(z)$ and

$$
\begin{array}{lrl}
q_{4}(z) & =16 z^{2}, & q_{8}(z)=3 z^{3}-46 z+24, \\
q_{5}(z)=-4 z\left(z^{3}+4 z^{2}-5 z+8\right), & q_{9}(z)=-3 z^{2}-8 z+17, \\
q_{6}(z)=-4\left(z^{4}+z^{3}-14 z^{2}+10 z-4\right), & q_{10}(z)=z+4 . \\
q_{7}(z)=-(z-1)\left(z^{3}-7 z^{2}-44 z+20\right), &
\end{array}
$$


Consider the nonnegative subharmonic function $w(z)=\sum_{n=4}^{10}\left|p_{n}(z)\right| d^{n}$ in the region $D=\{1-d \leq|z| \leq c, \quad 0.8 \leq \arg z \leq 2 \pi-0.8\} . \quad w(z)$ assumes its maximum on one of the four boundaries

$$
\begin{array}{ll}
B_{1}=\left\{c e^{i \phi}: 0.8 \leq \phi \leq 2 \pi-0.8\right\}, & B_{3}=\left\{x e^{i 0.8}: 1-d \leq x \leq c\right\}, \\
B_{2}=\left\{(1-d) e^{i \phi}: 0.8 \leq \phi \leq 2 \pi-0.8\right\}, & B_{4}=\left\{x e^{-i 0.8}: 1-d \leq x \leq c\right\} .
\end{array}
$$

It is easy to check that

$$
\begin{array}{rlrl}
\left|p_{10}\right| d^{10} & <1.210^{-5}, & & \left|p_{6}\right| d^{6}<7.410^{-2} \\
\left|p_{9}\right| d^{9} & <1.910^{-4}, & & \left|p_{5}\right| d^{5}<2.210^{-1} \\
\left|p_{8}\right| d^{8} & <2.410^{-3}, & & \left|p_{4}\right| d^{4}<6.2310^{-1} \\
\left|p_{7}\right| d^{7} & <1.710^{-2}, &
\end{array}
$$

for $z \in B_{1}$;

$$
\begin{array}{rlrl}
\left|p_{10}\right| d^{10} & <9.210^{-6}, & & \left|p_{6}\right| d^{6}<6.110^{-2}, \\
\left|p_{9}\right| d^{9} & <1.610^{-4}, & & \left|p_{5}\right| d^{5}<1.510^{-1} \\
\left|p_{8}\right| d^{8} & <1.910^{-3}, & & \left|p_{4}\right| d^{4}<3.410^{-1} \\
\left|p_{7}\right| d^{7} & <1.310^{-2}, &
\end{array}
$$

for $z \in B_{2}$; and

$$
\begin{array}{rlrl}
\left|p_{10}\right| d^{10} & <1.110^{-5}, & & \left|p_{6}\right| d^{6}<4.110^{-2} \\
\left|p_{9}\right| d^{9} & <1.610^{-4}, & & \left|p_{5}\right| d^{5}<1.210^{-1} \\
\left|p_{8}\right| d^{8} & <1.910^{-3}, & & \left|p_{4}\right| d^{4}<6.2310^{-1} \\
\left|p_{7}\right| d^{7} & <9.410^{-3}, &
\end{array}
$$

for $z \in B_{3} \cup B_{4}$. Thus

$$
|p(\gamma, \beta)| \leq \max _{z \in D} \sum_{n=4}^{10}\left|p_{n}(z)\right| d^{n}<0.94,
$$

and hence $0.94(m+\epsilon)>|\gamma p(\gamma, \beta)| \geq m$ by (3.4), a contradiction.

Therefore $m \geq d$. Let $\langle\phi, \psi\rangle$ denote the $(2,3,7)$ triangle group with $\phi^{2}=\psi^{3}=(\phi \psi)^{7}=\mathrm{id}$, and set $f=[\phi, \psi], h=\phi \psi, g=h f h^{-1}$. Then

$$
\begin{aligned}
\beta(f) & =\beta(g)=2(\cos (2 \pi / 7)+\cos (\pi / 7)-1)=c, \\
\gamma(f, g) & =\gamma(f, h)(\gamma(f, h)-\beta(f))=2 \cos (\pi / 7)-2=-d .
\end{aligned}
$$

3.8. Corollary. If $\langle f, g\rangle$ is discrete with $\gamma(f, g) \neq 0$ and $\gamma(f, g) \neq \beta(f)$ and if $|\beta(f)| \leq 2(\cos (2 \pi / 7)+\cos (\pi / 7)-1)=1.0489 \ldots$, then

$$
|\gamma(f, g)| \geq 2-2 \cos (\pi / 7)=0.198 \ldots .
$$

Inequality (3.9) is sharp.

Proof. We observe that $\gamma(f, g)=\gamma(f, f g)$. Thus if $g$ or $f g$ is not of order two, then (3.9) holds by Theorem 3.1. Suppose that $g$ and $f g$ are both of order two. Then $\beta\left(f g f g^{-1}\right)=\beta(f g f g)=0$. Since

$$
\beta\left(f g f g^{-1}\right)=(\beta(f)-\gamma(f, g))(\beta(f)-\gamma(f, g)+4),
$$


we have $\beta(f)-\gamma(f, g)+4=0$. Thus

$$
|\gamma(f, g)|=|\beta(f)+4| \geq 4-c,
$$

and hence (3.9) holds.

The example in Theorem 3.1 shows (3.9) is sharp.

\section{EQUAL TRACE PROBLEM}

4.1. Theorem. Suppose that $\langle f, g\rangle$ is a discrete subgroup of $\mathbb{M}$ with $\gamma(f, g) \neq$ 0 and $\beta(f)=\beta(g) \neq-4$. Then

$$
|\gamma(f, g)| \geq 2-2 \cos (\pi / 7)=0.198 \ldots
$$

Inequality (4.2) is sharp.

Proof. We use [4, Lemma 3.18]: For any discrete subgroup $\langle f, g\rangle$ with $\gamma(f, g)$ $\neq 0$ and $\beta(f)=\beta(g) \neq-4$, if

$$
\min \left\{|\beta(f)|,|\beta(f g)|,\left|\beta\left(f g^{-1}\right)\right|\right\} \geq c=2(\cos (2 \pi / 7)+\cos (\pi / 7)-1),
$$
then $|\gamma(f, g)| \geq d=2-2 \cos (\pi / 7)$.

If (4.3) holds, then (4.2) follows from [4, Lemma 3.18]. Otherwise, since

$$
\gamma(f, g)=\gamma(f g, g)=\gamma\left(f g^{-1}, g\right),
$$

we may assume by relabeling that $|\beta(f)|<c$. By assumption, $\beta(g) \neq-4$. Hence $|\gamma(f, g)| \geq d$ by Theorem 3.1.

Finally the example in Theorem 3.1 shows (4.2) is sharp.

\section{SOME CONSEQUeNCES OF THEOREM 4.1}

5.1. Theorem. Suppose that $\langle f, g\rangle$ is a discrete subgroup of $\mathbb{M}$ with $\gamma(f, g) \neq$ 0 and $\gamma(f, g) \neq \beta(f)$. If $f$ is not of order two, then

$$
\begin{aligned}
|\gamma(f, g)(\gamma(f, g)-\beta(f))| & \geq 2-2 \cos (\pi / 7)=0.198 \ldots, \\
|\gamma(f, g)(\beta(f)+4)| & \geq 2-2 \cos (\pi / 7)=0.198 \ldots, \\
|(\beta(f)-\gamma(f, g))(\beta(f)+4)| & \geq 2-2 \cos (\pi / 7)=0.198 \ldots .
\end{aligned}
$$

Each of these inequalities is sharp.

Proof. Consider the subgroup $\left\langle f, g f g^{-1}\right\rangle$.

$$
\beta\left(g f g^{-1}\right)=\beta(f) \neq-4, \quad \gamma\left(f, g f g^{-1}\right)=\gamma(f, g)(\gamma(f, g)-\beta(f)) \neq 0 .
$$

So, $\left|\gamma\left(f, g f g^{-1}\right)\right| \geq d=2-2 \cos (\pi / 7)$ by Theorem 4 .1. The example in [4, p. 210] shows (5.2) is sharp.

By [7, Lemma 2.29], there exists an elliptic $h$ of order 2 such that $\langle f, h\rangle$ is discrete and $\gamma(f, h)=\gamma(f, g)$. Thus

$$
\begin{aligned}
\beta(h f) & =\beta(f h)=\gamma(f, g)-\beta(f)-4 \neq-4, \\
\gamma(h f, f h) & =\gamma\left(f^{2}, h\right)=\gamma(f, h)(\beta(f)+4) \neq 0 .
\end{aligned}
$$

So, $|\gamma(h f, f h)| \geq d$ by Theorem 4.1. The example in [4, p. 210] and the fact that $\gamma(f h, h)(\beta(f h)+4)=\gamma(f, h)(\gamma(f, g)-\beta(f))$ show that (5.3) is sharp. 
By [7, Lemma 2.29], there exists an elliptic $\bar{h}$ of order 2 such that $\langle f, \bar{h}\rangle$ is discrete and $\gamma(f, \bar{h})=\beta(f)-\gamma(f, g)$. Thus (5.4) follows from (5.3). The example for (5.3) and the property of $\bar{h}$ show that (5.4) is sharp.

5.5. Remark. Many universal constraints for a discrete Möbius group $G$ are obtained by studying the sequence $\left\{\operatorname{tr}\left(\left[f, g_{n}\right]\right)\right\}$ where $f$ and $g_{1}$ are in $G$, $g_{n+1}=g_{n} f g_{n}^{-1}$. Among these results are the Shimizu-Leutbecher inequality, Jørgensen's inequality, and some variants in [3], [5], [7], [14], and [15]. It follows from (5.2) that

$$
\left|\operatorname{tr}\left[f, g_{n}\right]-2\right| \geq d, \quad \text { for all } n>1,
$$

in the above process. One example is the $(2,3,7)$ triangle group $\left\langle f, g_{1}\right\rangle$ for which $\gamma\left(f, g_{1}\right)=2 \cos (2 \pi / 7)-1, \beta(f)=c$. Thus

$$
\begin{array}{ll}
\operatorname{tr}\left[f, g_{n}\right]-2=2 \cos (\pi / 7)-2=-.198 \ldots, & \text { for } n=2 k, \\
\operatorname{tr}\left[f, g_{n}\right]-2=2 \cos (2 \pi / 7)-1=.2469 \ldots, & \text { for } n=2 k+1 .
\end{array}
$$

See [6] for more about the iterated commutators.

If $f$ is the square of some element in a discrete group, then $|\gamma(f, g)| \geq d$ by (5.3). For example, let $\langle f, g\rangle$ be a discrete group with $\gamma(f, g) \neq 0$. The Lie product of $f$ and $g$ defines a Möbius transformation $\phi$ which is elliptic of order two. The mapping $\phi f^{-1} g^{-1}$ is a square root of $f g f^{-1} g^{-1}$ [9, Section 4]. By (5.2), $\left|\gamma\left(f g f^{-1} g^{-1}, f\right)\right|=|\gamma(f, g)(\gamma(f, g)-\beta(f))| \geq d$.

5.6. Theorem. Suppose that $\langle f, g\rangle$ is discrete with $\gamma(f, g) \neq 0$.

If $\beta(f) \neq-1$, then $|\gamma(f, g)|+|\beta(f)+1| \geq c_{1}, \quad .426<c_{1} \leq .493 \ldots$.

If $\beta(f) \neq-2$, then $|\gamma(f, g)|+|\beta(f)+2| \geq c_{2}, \quad .806<c_{2} \leq 1$.

If $\beta(f) \neq-3$, then $|\gamma(f, g)|+|\beta(f)+3| \geq c_{3}, .908<c_{3} \leq 1$.

If $\beta(f) \neq-4$, then $|\gamma(f, g)|+|\beta(f)+4| \geq c_{4}, .890<c_{4} \leq 1.048 \ldots$.

Proof. Let $\gamma=\gamma(f, g), \beta=\beta(f)$. It follows from (5.3) and the ArithmeticGeometric Mean inequality that $c_{4} \geq 2 \sqrt{d}=.89 \ldots$ If we replace $f$ by $f^{2}$ in (5.3) and minimize $|\gamma|+|\beta+2|$ subject to the constraint

$$
\left|\gamma\left(f^{2}, g\right)\left(\beta\left(f^{2}\right)+4\right)\right|=\left|\gamma(\beta+4)(\beta+2)^{2}\right| \geq d,
$$

we get $c_{2}>.806$. Next we replace $f$ by $f^{3}$ in (5.2) and (5.3). Minimizing $|\gamma|+|\beta+3|$ subject to the constraint

$$
\left|\gamma\left(f^{3}, g\right)\left(\gamma\left(f^{3}, g\right)-\beta\left(f^{3}\right)\right)\right|=\left|\gamma(\beta+3)^{4}(\gamma-\beta)\right| \geq d
$$

gives $c_{3}>.908$. Minimizing $|\gamma|+|\beta+1|$ subject to the constraint

$$
\left|\gamma\left(f^{3}, g\right)\left(\beta\left(f^{3}\right)+4\right)\right|=\left|\gamma(\beta+3)^{2}(\beta+4)(\beta+1)^{2}\right| \geq d
$$

gives $c_{1}>.426$.

We now give the upper bounds. Since $\gamma_{1}=2 \cos (2 \pi / 7)-1, \beta_{1}=\gamma_{1}-1$, $\beta_{1}^{\prime}=-4$ are discrete parameters, $c_{1} \leq 2(2 \cos (2 \pi / 7)-1)=.493 \ldots$. By [10], for $1<a<\infty, \gamma_{3}=4(a-1 / a)^{-2}, \beta_{3}=-4, \beta_{3}^{\prime}=-(a+1 / a)^{2}$ are discrete parameters. Thus $\left|\gamma_{3}\right|+\left|\beta_{3}+3\right| \rightarrow 1$ as $a \rightarrow \infty$. Hence $c_{3} \leq 1$. Since $\beta_{2}=(\sqrt{5}-5) / 2, \quad \gamma_{2}=\beta_{2}+1, \beta_{2}^{\prime}=-4$ are discrete parameters, $c_{2} \leq 1$. Note that $\gamma=2 \cos (2 \pi / 7)-1, \beta=c, \beta^{\prime}=c$ are discrete parameters. So 
$\gamma_{4}=2 \cos (2 \pi / 7)-1, \beta_{4}=\gamma-\beta-4, \beta_{4}^{\prime}=-4$ are discrete parameters. Thus $c_{4} \leq\left|\gamma_{4}\right|+\left|\beta_{4}+4\right|=c=1.048 \ldots$.

5.7. Remark. There are some similar results in [14] and [15]. Gehring and Martin have shown that $|\gamma(f, g)|+|\beta(f)+1| \geq 1$ if $\gamma(f, g) \neq 0, \beta(f) \neq-1$, and $\gamma(f, g) \neq \beta(f)+1$.

5.8. Lemma. Suppose that $A$ and $B$ generate a discrete nonelementary subgroup of $\mathrm{SL}(2, \mathbb{C})$. Then

$$
\|A-I\|\|B-I\|>k, \quad .46<k \leq .52 \ldots .
$$

Proof. For any $C \in \mathrm{SL}(2, \mathbb{C})$, we define $m(C)=\left\|C-C^{-1}\right\|$ where $\|\cdot\|$ is the usual Hilbert-Schmidt norm of a matrix (see [5]). Then

$$
\begin{aligned}
4\|C-I\|^{2}= & 2|\operatorname{tr}(C)-2|^{2}+m^{2}(C), \\
\left\|A-\left.I\right|^{2}\right\| B-\left.I\right|^{2}= & \frac{1}{4}|\operatorname{tr}(A)-2|^{2}|\operatorname{tr}(B)-2|^{2}+\frac{1}{8}|\operatorname{tr}(A)-2|^{2} m^{2}(B) \\
& +\frac{1}{8}|\operatorname{tr}(B)-2|^{2} m^{2}(A)+\frac{1}{16} m^{2}(A) m^{2}(B) .
\end{aligned}
$$

Let $x=\min \left\{\left|\operatorname{tr}^{2}(A)-4\right|,\left|\operatorname{tr}^{2}(B)-4\right|\right\}$. By [5, Theorem 2.7],

$$
m^{2}(C) \geq 2\left|\operatorname{tr}^{2}(C)-4\right|, \quad m^{2}(A) m^{2}(B) \geq 16|\operatorname{tr}[A, B]-2| .
$$

If $x \leq 0.8$, then $\|A-I\|\|B-I\|>0.46$ by (5.9) and Jørgensen's inequality. If $0.8 \leq x \leq c$, then we replace $m^{2}(A) m^{2}(B)$ by $16 d$ and get $\|A-I\|\|B-I\|>$ 0.46 . If $x \geq c$, then $\|A-I\|\|B-I\|>0.46$ by (5.9).

Let $\langle\phi, \psi\rangle$ denote the $(2,3,7)$ triangle group with $\phi^{2}=\psi^{3}=(\phi \psi)^{7}=$ id . The transformations $\phi$ and $\psi$ can be represented by the matrices

$$
A=\frac{i}{\sin a}\left(\begin{array}{cc}
-\cos b & -p \\
p & \cos b
\end{array}\right), \quad B=\left(\begin{array}{cc}
e^{i a} & 0 \\
0 & e^{-i a}
\end{array}\right),
$$

where $a=\pi / 3, b=\pi / 7$ and $p=\left(\cos ^{2} b-\sin ^{2} a\right)^{1 / 2}[12$, p. 88]. We set $C=[A, B]$ and $D=A B$. Then

$$
\gamma(C, D)=2 \cos (2 \pi / 7)-1, \quad \beta(C)=c, \quad \beta(D)=2 \cos (2 \pi / 7)-2 .
$$

We can find a Möbius transformation $h$ which sends the fixed points of $C$ to $\{w,-w\}$ and sends the fixed points of $D$ to $\{1 / w,-1 / w\}$. By [5, Lemma 2.12 ], such a $w$ satisfies the equation

$$
\left(w^{2}-1 / w^{2}\right)^{2}=16 \frac{\gamma(C, D)}{\beta(C) \beta(D)} .
$$

Let $u=|w|^{2}+1 /|w|^{2}$ and $v=2 \cos (2 \pi / 7)-1$. Then $m^{2}\left(h C h^{-1}\right)=u \beta(C)$ and $m^{2}\left(h D h^{-1}\right)=-u \beta(D)$ by [5, Lemma 2.10]. Therefore,

$$
\begin{aligned}
& \left\|h C h^{-1}-I\right\|\left\|h D h^{-1}-I\right\| \\
& =\left(v+\frac{1}{4} v^{2} d^{2}+\frac{1}{2}\left(v^{2}-v^{3}+c d^{2}\right) \sqrt{v / c(1-v)}\right)^{1 / 2} \\
& =.5214 \ldots .
\end{aligned}
$$

5.10. Remark. Waterman has shown that $\|A-I\|\|B-I\|>\sqrt{2}-1$ by means of Jørgensen's inequality [16]. 
Set $E=B C B^{-1}$. By [5, Lemma 2.27], there exists an $h$ in $\mathbb{M}$ such that $m^{2}\left(h C h^{-1}\right)=2 \beta(C)$ and $m^{2}\left(h E h^{-1}\right)=2 \beta(E)$. So $\left\|h C h^{-1}-I\right\|=$ $\left\|h E h^{-1}-I\right\|=\left(\left(v^{2}+c\right) / 2\right)^{1 / 2}=.7449 \ldots$ Therefore, if $\langle A, B\rangle$ is a discrete nonelementary subgroup of $\operatorname{SL}(2, \mathbb{C})$, then

$$
\max \{\|A-I\|,\|B-I\|\}>t, \quad .67<t \leq .74 \ldots .
$$

\section{ACKNOWLEDGMENT}

The author wishes to thank Professor F. W. Gehring for suggesting this topic and for many helpful conversations. He also thanks the Department of Mathematics of the University of Auckland for its hospitality while this paper was being written.

\section{REFERENCES}

1. C. Cao, On three generator Möbius groups, New Zealand J. Math. 23 (1994), 111-120.

2. F. W. Gehring and G. J. Martin, Stability and extremality in Jørgensen's inequality, Complex Variables Theory Appl. 12 (1989), 277-282.

3. __ Iteration theorey and inequalities for Kleinian groups, Bull. Amer. Math. Soc. (N.S.) 21 (1989), 57-63.

4. __ Some universal constraints for discrete Möbius groups, Paul Halmos; Celebrating 50 Years of Mathematics, Springer-Verlag, New York, pp. 205-220.

5. (1991), 31-76.

6. The iterated commutator in a Fuchsian group, Complex Variables Theory Appl. 21 (1993), 207-218.

7. _ Commutators, collars and the geometry of Möbius groups, J. Analyse Math. 63 (1994), $175-219$.

8. T. Jørgensen, On discrete groups of Möbius transformations, Amer. J. Math. 98 (1976), 739-749.

9. Compact 3-manifolds of constant negative curvature fibering over the circle, Ann. of Math. (2) 106 (1977), 61-72.

10. Comments on a discreteness condition for subgroups of $\operatorname{SL}(2, \mathbb{C})$, Canad. J. Math. 31 (1979), 87-92.

11. _ Commutators in SL $(2, \mathbb{C})$, Riemann Surfaces and Related Topics: Proceedings of the 1978 Stony Brook Conference, Princeton Univ. Press, Princeton, NJ, 1980, pp. 301-303.

12. W. Magnus, Noneuclidean tesselations and their groups, Academic Press, Boston, MA, 1974.

13. B. Maskit, Kleinian groups, Springer-Verlag, New York, 1988.

14. D. Tan, On two-generator discrete groups of Möbius transformations, Proc. Amer. Math. Soc. 106 (1989), 763-770.

15. __ On inequalities for discrete groups of Möbius transformations (to appear).

16. P. Waterman, An inscribed ball for Kleinian groups, Bull. London Math. Soc. 16 (1984), 525-530.

Department of Mathematics, University of Michigan, Ann Arbor, Michigan 48109

E-mail address: ccao@math.lsa.umich.edu 